\title{
Recombination Reactions of Atomic Chlorine in Inert Gases: A Vibrationally Resolved Transient Kinetics Study at Pressures below 1 atm
}

\author{
L.-C. Chang, Y.-S. Hwang, and T.-M. Su* \\ Department of Chemistry, National Taiwan University, Taipei, Taiwan, R.O.C., \\ and The Institute of the Atomic and Molecular Sciences, Academia Sinica, Taipei, Taiwan, R.O.C. \\ Received: November 24, 1997; In Final Form: February 18, 1998
}

\begin{abstract}
The chlorine atom recombination reactions in the buffer gases of chlorine, argon, nitrogen, and carbon dioxide below 1 atm pressure were studied by the laser photolysis/chemiluminescence detection technique. The vibrationally resolved transient chemiluminescence spectra were recorded. A reaction mechanism which included recombinations, electronic quenching, and vibrational energy relaxation processes of both the A and $\mathrm{B}$ excited states of the chlorine molecules was proposed to account for the present experimental observations and also the related experimental results reported in the literature. Under the approximation of the exponential energy-gap rate laws for the rate constants of the inter- and intraelectronic vibrational energy relaxations as proposed in the literature, either the rate constants of electronic quenching, vibrational relaxation within the same electronic states, and interelectronic vibrational energy transfer between the A and B states of the chlorine molecules by the buffer gases were determined or their upperbound values were estimated. The long-time steady state behavior of the mechanism was also analyzed analytically and numerically.
\end{abstract}

\section{Introduction}

The recombination reactions of the chlorine atoms and their accompanied physical processes, such as the electronic quenching and the vibrational energy relaxation in the electronic excited states, have been investigated by a number of research groups since the $1960 \mathrm{~s} .{ }^{1-14}$ The rate constants of the recombination, the photon emission of the B state of the chlorine molecule, and the electronic quenching by some third bodies have been measured by a variety of techniques over a quite wide pressure range. Nevertheless, it was only quite recently that the chemiluminescence of the recombination reactions was known quantitatively to be composed of the emissions of the B and A states instead of the pure B state as traditionally assumed. ${ }^{12}$ Evidently, a reexamination of the traditionally accepted reaction mechanism for the radiative recombination of the chlorine atoms is needed. Additionally, since the major vibrational states involved could be resolved quantitatively under the present experimental conditions, for the first time, the vibrationally resolved transient luminescence during the recombination reaction could be taken and the time-evolution of the vibrational state populations could be analyzed systematically.

In this paper, measurements of the vibrationally resolved transient luminescence of the chlorine atom recombination reaction with chlorine, argon, nitrogen, and carbon dioxide gases as third bodies were reported. Along with the related measurements available in the literature, a more comprehensive reaction mechanism for the radiative recombination of the chlorine atoms was proposed. Through a full simulation of the experimental results, either the general rate constants of the vibrational energy relaxation, vibrational energy exchange between the $\mathrm{A}$ and $\mathrm{B}$ states, and other processes related with the recombination reactions were obtained or their upperbound values were estimated. Additionally, the present study provided a kinetic interpretation for the apparent electronic quenching rate constants of the B state reported in the literature and also an experimental estimation for the extent of the population over the vibrationally excited states at the initial atomic chlorine recombination stage.

\section{Experimental Section}

The basic experimental setup was similar to those reported previously in the measurements of the atomic recombination chemiluminescence spectra and the halogen atom diffusion coefficients. ${ }^{12,15}$ Some modifications were made to meet the specific requirements of the present experiment. Figure 1 shows the schematic diagram of the experimental arrangement. The photolysis light was $355 \mathrm{~nm}$ wavelength, $5 \mathrm{~ns}$ duration pulses of a Q-switched Nd:YAG laser. The main body of the flow reactor was constructed from a stainless steel cube of $15 \mathrm{~cm}$ for each side. Light baffles made from black graphitized Teflon were placed inside the reaction cell to reduce the laser scattering. Through a side window, the chemiluminescence was focused into a 275-mm focal length monochromator with a reciprocal linear dispersion of $3 \mathrm{~nm} / \mathrm{mm}$. Depending on the vibrational bands, a slit width varied from 1.0 to $2.0 \mathrm{~mm}$ was used. The photomultiplier signals were processed by a multichannel scaler with a dwell time of $200 \mathrm{~ns}$. For the few experimental situations with the weakest signals, an average over around one million laser shots was needed to enhance the $\mathrm{S} / \mathrm{N}$ ratio.

The sample cell was kept at $293 \mathrm{~K}$ by a thermostat. The chlorine (natural isotope abundance; semiconductor grade, 99.997\%; Scott Specialty Gases), argon (99.9995\%; Scott Specialty Gases), nitrogen (99.9995\%; Spectra Gases), and carbon dioxide (SFC grade, $>99.9999 \%$; Air Products) were used directly from gas cylinders without further purification. The highest chlorine pressure used was 5.33 mbar and the highest inert gas pressure used was 264 mbar. The laser energy was monitored by a calibrated pyroelectric joule meter and kept at a level such that the chlorine atom concentration at the center of the reaction cell was around $5.2 \times 10^{13}$ molecules $/ \mathrm{cm}^{3}$. 


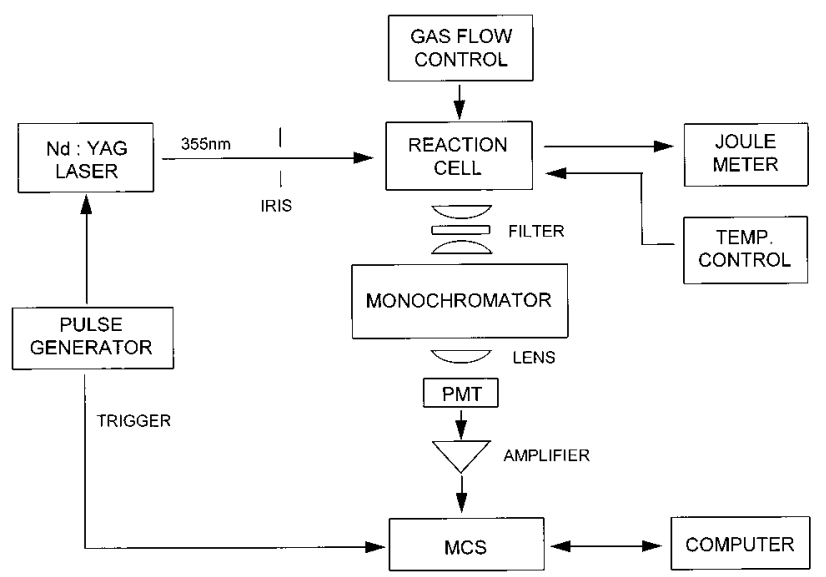

Figure 1. Schematic diagram of the experimental setup. MCS: multichannel scaler. PMT: photomultiplier tube.

\section{Kinetic Model}

The chemiluminescence reaction mechanism of the photolysis/recombination processes of chlorine molecules under the radiation of a short 355-nm laser pulse in an inert buffer gas $\mathrm{M}$ could be adequately represented by $9,12,13,16$

$$
\begin{gathered}
\mathrm{Cl}_{2}+h v \stackrel{\text { photodissociation }}{\longrightarrow} 2 \mathrm{Cl} \\
\mathrm{Cl}+\mathrm{Cl}+\mathrm{M} \underset{D_{v}^{\mathrm{B}}}{\stackrel{R_{u}^{\mathrm{B}}}{\leftrightarrows}} \mathrm{Cl}_{2} *(\mathrm{~B}, v)+\mathrm{M} \\
\mathrm{Cl}+\mathrm{Cl}+\mathrm{M} \underset{D_{u}^{\mathrm{A}}}{\stackrel{R_{u}^{\mathrm{A}}}{\leftrightarrows}} \mathrm{Cl}_{2} *(\mathrm{~A}, u)+\mathrm{M} \\
\mathrm{Cl}_{2} *(\mathrm{~B}, v)+\mathrm{M} \underset{V_{i v}^{\mathrm{B}}}{\stackrel{V_{v i}^{\mathrm{B}}}{\rightleftarrows}} \mathrm{Cl}_{2} *(\mathrm{~B}, i)+\mathrm{M} \\
\mathrm{Cl}_{2} *(\mathrm{~A}, u)+\mathrm{M} \underset{V_{j u}^{\mathrm{A}}}{\stackrel{V_{u j}^{\mathrm{A}}}{\leftrightarrows}} \mathrm{Cl}_{2} *(\mathrm{~A}, j)+\mathrm{M} \\
\mathrm{Cl}_{2} *(\mathrm{~B}, v)+\mathrm{M} \underset{k_{u v}^{\mathrm{A}}}{\stackrel{k_{v u}^{\mathrm{B}}}{\longrightarrow}} \mathrm{Cl}_{2} *(\mathrm{~A}, u)+\mathrm{M} \\
\mathrm{Cl}_{2} *(\mathrm{~B}, \mathrm{~A})+\mathrm{M} \stackrel{Q_{\mathrm{B}} \text { and } Q_{\mathrm{A}}}{\longrightarrow} \mathrm{Cl}_{2}(\text { nonradiative })+\mathrm{M} \\
\mathrm{Cl}_{2} *(\mathrm{~B}, \mathrm{~A}, v) \stackrel{e_{v}^{\mathrm{B}} \text { and } e_{u}^{\mathrm{A}}}{\longrightarrow} \mathrm{Cl}_{2}(\mathrm{X})+h v
\end{gathered}
$$

Here, $\mathrm{Cl}_{2} *(\mathrm{~A}, u)$ and $\mathrm{Cl}_{2} *(\mathrm{~B}, v)$ are vibrationally resolved $\mathrm{A}$ and $\mathrm{B}$ electronic states of $\mathrm{Cl}_{2}, \mathrm{M}$ is the third body which may be generalized to include any number of the chemical species in the system, $R_{v}$ and $D_{v}$ are the recombination and dissociation rate constants of the $v$ vibrational state, respectively, $V_{u i}$ 's and $V_{v i}$ 's are the vibrational energy transfer rate constants within the $\mathrm{A}$ and $\mathrm{B}$ electronic states themselves, respectively, $k_{v u}$ is the vibrational energy exchange rate constant between the $\mathrm{A}$ and B electronic state, $Q$ s are the average electronic quenching rate constants, and $e$ 's are the rate constants of photon emission. The superscripts or subscripts of A and B appearing in the rate constants specify the A and B electronic states, respectively. The transient vibrationally resolved emission bands due to the process of eq 8 were detected in the present experiment.

The nonpredissociated vibrational energy levels of the B state and all the bound vibrational energy levels of the A state were considered in the above mechanism. As shown in Figure 2, 13 lowest vibrational energy levels of the B state which have been well characterized are involved. ${ }^{9,17}$ For the A state, the lowest

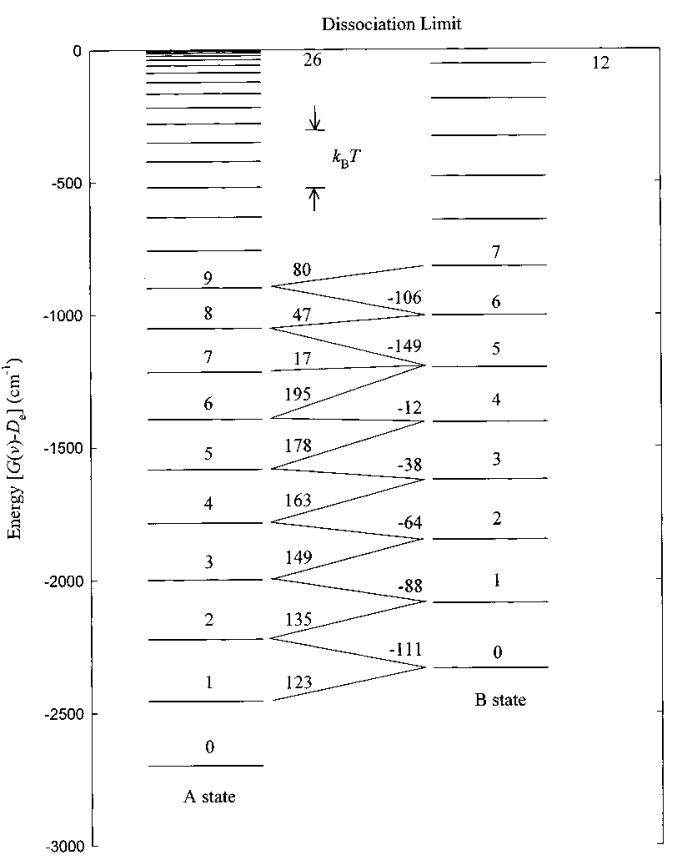

Figure 2. Vibrational energy levels of the $\mathrm{A}$ and $\mathrm{B}$ states of $\mathrm{Cl}_{2}$. Some selected energy differences between the $\mathrm{A}$ and $\mathrm{B}$ vibrational states are also indicated. In the present experiments, $k_{\mathrm{B}} T=208 \mathrm{~cm}^{-1}$.

13 vibrational energy levels have been determined by a recent spectroscopic study, and their highest energy level is located at $421.7 \mathrm{~cm}^{-1}$ below the dissociation energy. ${ }^{18}$ The remaining vibrational energy levels near the dissociation limit were then theoretically calculated by the Le Roy-Bernstein method as follows. ${ }^{19}$ To begin with, the long-range interaction potential was assumed to follow the functional form of $-C_{n} / r^{n}$. The $n$ value was obtained to be 6.9 by fitting the potential to the six highest outer turning points of the RKR potential. ${ }^{18}$ The extrapolated vibrational energies were then calculated according to the relation

$$
\left[D_{\mathrm{e}}-G(v)\right]^{(n-2) / 2 n}=\left(v_{\mathrm{d}}-v\right) H_{n}
$$

in which $D_{\mathrm{e}}$ is the dissociation energy and $G(v)$ is the vibrational energy. The constants $v_{\mathrm{d}}$ and $H_{n}$ were determined to be 26.05 and 0.656 , respectively, through least-squares fitting to the six highest experimental vibrational energies. A total of 27 bound vibrational states was obtained in the A state. Figure 2 shows the complete relative energies of the vibrational states considered in this report. The master equation which describes the vibrationally resolved recombination mechanism of eqs $2-8$ can be written as

$$
\begin{array}{r}
\frac{\mathrm{d} B_{v}}{\mathrm{~d} t}=R_{v}^{\mathrm{B}} M[\mathrm{Cl}]^{2}+\sum_{i=0}^{12} V_{i v}^{\mathrm{B}} B_{i} M+\sum_{u=0}^{26} k_{u v}^{\mathrm{A}} A_{u} M-\left(D_{v}^{\mathrm{B}} B_{v} M+\right. \\
\left.\sum_{i=0}^{12} V_{v i}^{\mathrm{B}} B_{v} M+\sum_{u=0}^{26} k_{v u}^{\mathrm{B}} B_{v} M+Q_{\mathrm{B}} B_{v} M+e_{v}^{\mathrm{B}} B_{v}\right)
\end{array}
$$

$$
\begin{array}{r}
\frac{\mathrm{d} A_{u}}{\mathrm{~d} t}=R_{u}^{\mathrm{A}} M[\mathrm{Cl}]^{2}+\sum_{v=0}^{12} k_{v u}^{\mathrm{B}} B_{v} M+\sum_{j=0}^{26} V_{j u}^{\mathrm{A}} A_{j} M-\left(D_{u}^{\mathrm{A}} A_{u} M+\right. \\
\left.\sum_{j=0}^{26} V_{u j}^{\mathrm{A}} A_{u} M+\sum_{v=0}^{12} k_{u v}^{\mathrm{A}} A_{u} M+Q_{\mathrm{A}} A_{u} M+e_{u}^{\mathrm{A}} A_{u}\right)
\end{array}
$$

in which $B_{v}$ and $A_{u}$ are the vibrational populations of the $\mathrm{B}$ and A states, respectively, $v$ covers values from 0 to 12 for the $\mathrm{B}$ 
state, and $u$ covers values from 0 to 26 for the A state. Since in the above equations, the vibrationally resolved independent rate constants still outnumbered the experimental measurements available even if the relations of the detailed balance were counted, the variations of the rate constants over the vibrational states for the recombination, vibrational energy relaxation, and vibrational energy exchange between the $\mathrm{A}$ and $\mathrm{B}$ states were further approximated by the following models.

As suggested in the results of the classical trajectory studies on the iodine and bromine atom recombination reactions, the recombination rate constants into the vibrational states of the A and B states could be approximated by the energy-gap exponential form: ${ }^{20,21}$

$$
\begin{aligned}
& R_{v}^{\mathrm{B}}=R_{\mathrm{B}} e^{-\left[D_{\mathrm{e}}^{\mathrm{B}}-G(v)\right] / \gamma} / N_{\mathrm{B}} \\
& R_{u}^{\mathrm{A}}=R_{\mathrm{A}} e^{-\left[D_{\mathrm{e}}^{\mathrm{A}}-G(u)\right] / \gamma} / N_{\mathrm{A}}
\end{aligned}
$$

in which $D_{\mathrm{e}}^{\mathrm{A}}, D_{\mathrm{e}}^{\mathrm{B}}, R_{\mathrm{A}}$, and $R_{\mathrm{B}}$ are the equilibrium bond dissociation energies and the recombination rate constants of the A and B states, respectively, and $\gamma$ is a fitted parameter which is assumed to be the same for both states. The normalization constants $N_{\mathrm{A}}$ and $N_{\mathrm{B}}$ are defined as

$$
\begin{aligned}
& N_{\mathrm{B}}=\sum_{v=0}^{12} \exp \left[-\left(D_{\mathrm{e}}^{\mathrm{B}}-G(v)\right) / \gamma\right] \\
& N_{\mathrm{A}}=\sum_{u=0}^{26} \exp \left[-\left(D_{\mathrm{e}}^{\mathrm{A}}-G(u)\right) / \gamma\right]
\end{aligned}
$$

The corresponding dissociation rate constants were calculated by the principle of detailed balance:

$$
K_{\mathrm{eq}}(u)=R_{u} / D_{u}=q_{\mathrm{Cl}_{2} *(u)} L / q_{\mathrm{Cl}}^{2}
$$

in which the equilibrium constant of the vibrational $u$ state with respect to the dissociated state is calculated by the partition functions of $\mathrm{Cl}_{2} *(u)$ and $\mathrm{Cl}, q_{\mathrm{Cl}_{2} * u}$ and $q_{\mathrm{cl}}$, respectively, and $L$ is the volume of reaction cell. The collisional dissociation rate constant for a specific vibrational state $u$ in the units of $\mathrm{cm}^{3}$ molecule ${ }^{-1} \mathrm{~s}^{-1}$ becomes

$$
D_{u}^{\mathrm{A}, \mathrm{B}}=\left(1.78 \times 10^{24}\right) e^{-\left[D_{\mathrm{e}}^{\mathrm{A}, \mathrm{B}}-G(u)\right] / k_{\mathrm{B}} T} R_{u} / g
$$

in which the numerical factor is in the units of molecule $\mathrm{cm}^{-3}$ and $g$ is the electronic degeneracy and equals 2 for the A state and 1 for the B state. Since the moment of inertia of the A and B states are close to each other to within three significant numbers, in eq 17, a common conversion factor was employed for both states.

The vibrational energy deactivation rate constants within a specific electronic state are assumed to follow the simple exponential rate law $^{16,22,23}$

$$
V_{v i}=V_{0} \exp [-(G(v)-G(i)) / \alpha]
$$

in which $V_{0}$ and $\alpha$ are constants and the vibrational energy of level $v$ is assumed to be higher than that of level $i$. The vibrational activation rate constants were calculated according to the principle of detailed balance:

$$
V_{i v}=V_{v i} \exp \left[(G(i)-G(v)) / k_{\mathrm{B}} T\right]
$$

where $k_{\mathrm{B}}$ is the Boltzmann constant. For the present case, $V_{0}$ is the collision limit rate constant and is fixed at $3.2 \times 10^{-10}$ $\mathrm{cm}^{3}$ molecule ${ }^{-1} \mathrm{~s}^{-1}$, the hard-sphere bimolecular collision rate constant between $\mathrm{Cl}_{2}(\mathrm{~B})$ and $\mathrm{Cl}_{2}(\mathrm{X}) .{ }^{16,24}$ Since the A and B states belong to the same triplet state manifold with similar bonding strengths, their vibrational relaxation parameters are assumed to be the same in the present study.

For the vibrational energy transfer rate constants between the A and B states, the following exponential gap law was adapted ${ }^{25}$

$$
k_{v u}=\kappa k_{e x} \exp [-(G(v)-G(u)) / \beta]
$$

in which $k_{\text {ex }}$ and $\beta$ are fitted parameters, $\kappa$ is the multiplicity parameter which is equal to 1 for the $\mathrm{A}$ to $\mathrm{B}$ transition and 2 for the reverse process, and $G(v)>G(u)$. Since for the A and $\mathrm{B}$ states of $\mathrm{Cl}_{2}$ the major energy exchange process is expected to occur under near-resonant conditions as shown in the energy levels of Figure 2, the Boltzmann population factor would be a dominant factor under the present thermal equilibrium environment. In the following analysis, the $\beta$ value was further approximated by $k_{\mathrm{B}} T$. The reverse rate constants $k_{u v}$ were then calculated by the principle of detailed balance.

Under the present experimental conditions, the recombination rate of the chlorine atoms is about three order of the magnitude slower than the quenching and vibrational relaxation rates. In the time domain the present experiments are interested, the chlorine atom concentration is essentially a constant. The first nonlinear term of chlorine atom concentration in eqs (10) and (11) was approximated by a constant as a source term. The complete solutions of the final coupled first-order differential equations were then solved numerically by the variable-order variable-step Adams method adapted directly from a commercial Fortran library. ${ }^{26}$

\section{Results and Discussion}

Four buffer gases were used in the present study. The transient vibrationally resolved luminescences were recorded from $v=0$ to 5 for the B state and 0 and 1 for the A state. The signals of the remaining higher vibrational states were all too weak to have a reasonably good $\mathrm{S} / \mathrm{N}$ ratio. The measurements reported in the literature which are relevant to the present study were employed as constraints in the following simulation: the steady-state chemiluminescence spectra which correspond to the steady-state relative vibrational populations of the A and B states at the steady state and the total steady-state population ratio between the $\mathrm{A}$ and $\mathrm{B}$ states as determined by the spectral intensity distributions; ${ }^{12}$ the apparent electronic quenching rate constants of the B state by the chlorine gas as measured by the laser-induced fluorescence technique; ${ }^{16}$ the radiative state quenching rate constants of argon and nitrogen gases measured by the high-pressure transient recombination experiments; ${ }^{13}$ the steady-state low-pressure-limited photon-emission quantum efficiencies of the $\mathrm{A}$ and $\mathrm{B}$ states in the atomic chlorine recombination reactions with the argon buffer gas as determined by the chlorine afterglow spectra and revised in a recent study. ${ }^{9,12}$ With these experimental constraints, in the case of the neat chlorine system, the present transient measurements along with the steady-state emission spectra were simulated by solving the above 40 first-order coupled differential equations. For the cases of the other buffer gases, since there was always some fixed amount of chlorine molecules present in the experimental cell, an extended mechanism which consisted of two buffer gases was employed. By fixing those parameters obtained in the neat chlorine system, the equations were then solved numerically and the related rate constants of the buffer gases were determined in a similar way. 

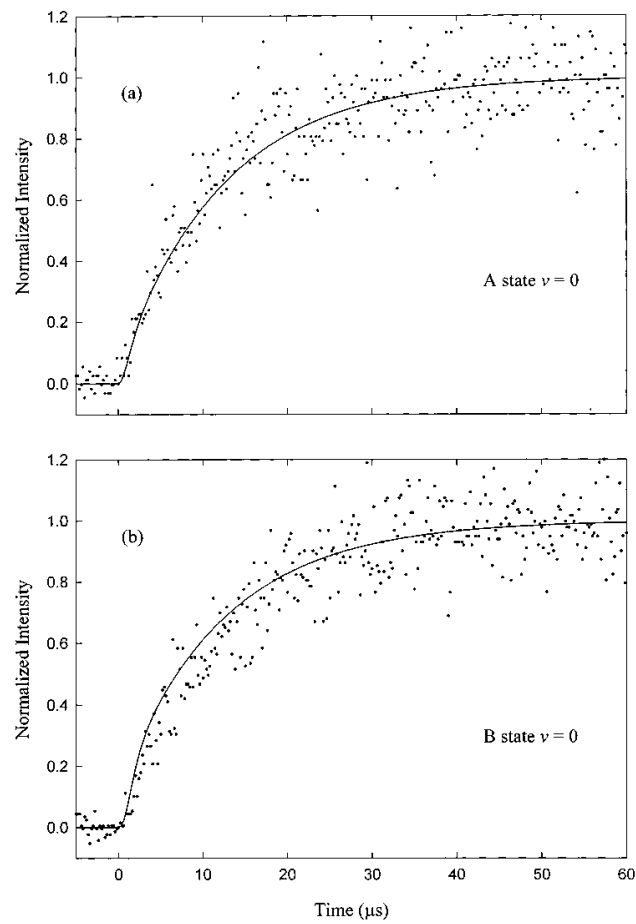

Figure 3. Transient recombination luminescence signals detected at $870.6 \mathrm{~nm}(\mathrm{~A}(v=0) \rightarrow \mathrm{X}(v=11))$ and $879.3 \mathrm{~nm}(\mathrm{~B}(v=0) \rightarrow \mathrm{X}(v=12))$, in neat $5.33 \mathrm{mbar} \mathrm{Cl}_{2}$. The smooth curves are the full simulation of the corresponding experimental conditions, which include the minor pickups of the luminescence wings from nearby vibrational bands by the detection system. The signals are normalized to the asymptotic state intensities.

4A. Neat Chlorine System. Figures 3 and 4 show some representative experimental transient signals detected at various vibrational bands in neat 5.33 mbar chlorine gas. To simulate the experiments, in an initial attempt, the A state emission was deliberately ignored and the conventional reaction mechanism which consists purely of the recombination, vibrational relaxation, and electronic quenching of the B state was considered. It turned out that under the conventional B state mechanism there were no ways that one could reconcile the inconsistency between the long-time decay rate constants of the present transient observations and the quenching rate constants of the B state measured by the laser induced fluorescence technique. ${ }^{9}$ The latter quantity is about 10 times the former one. It was concluded that the simplified mechanism was not adequate to account for all the available experimental results. As described in the previous section, a natural expanded mechanism which included the A state and the coupling between the A and B states was proposed in this report.

In the numerical simulation, it was further found that under the present experimental conditions the absolute recombination rate constants of $R_{\mathrm{A}}$ and $R_{\mathrm{B}}$ are not sensitive to the transient recombination signals within the experimental uncertainties and the related measurements. It was the relative ratio $R_{\mathrm{B}} / R_{\mathrm{A}}$ which was crucial to the simulation. To the authors' knowledge, to date there are no experimental $R_{\mathrm{A}}$ values available in the literature. Nevertheless, theoretically, by taking the electronic degeneracies into account, the A state recombination quantum yield has been calculated to be $34 \%$ by the energy transfer model in this laboratory. ${ }^{14}$ For the present purpose, $R_{\mathrm{A}}$ was directly set at the value of $34 \%$ of the total experimental recombination rate constant. It was found that a scaling of the theoretical $\mathrm{A}$ state recombination rate constant by a factor of 2 or $1 / 2-$ a range wide enough to cover the possible uncertainty of the above
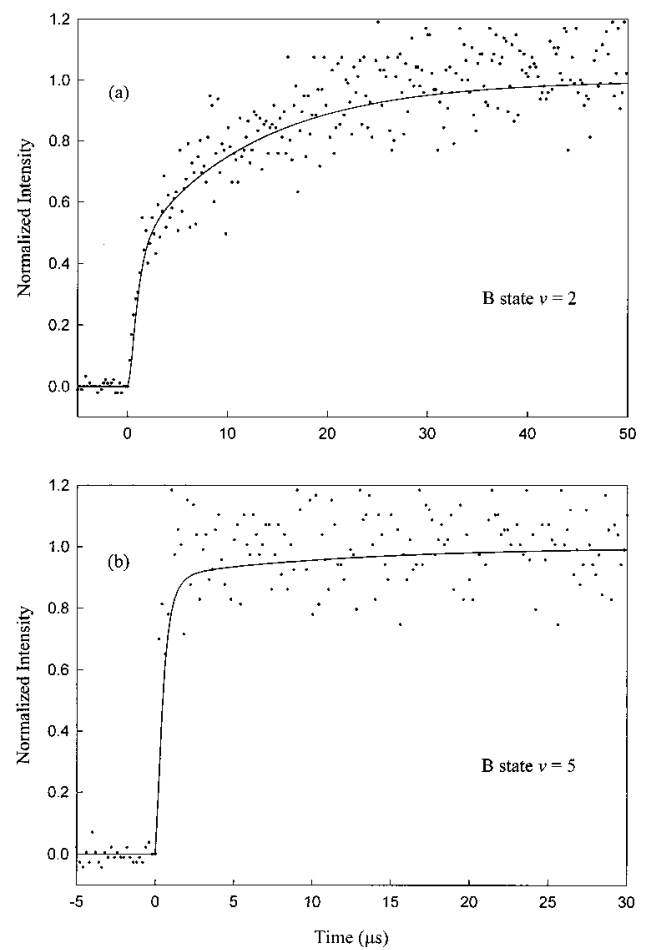

Figure 4. Transient recombination luminescence signals detected at $722.7 \mathrm{~nm}(\mathrm{~B}(v=2) \rightarrow \mathrm{X}(v=8))$ and $623.5 \mathrm{~nm}(\mathrm{~B}(v=5) \rightarrow \mathrm{X}(v=5))$, in neat $5.33 \mathrm{mbar} \mathrm{Cl}_{2}$. Since there was always some scattered light recorded at time zero as the detection wavelength was shorter than $700 \mathrm{~nm}$, the scattering signal recorded on the laser-flashing channel has been reset to the background level in (b). The smooth curves are the full simulation of the corresponding experimental conditions, which include the minor pickups of the luminescence wings from nearby vibrational bands by the detection system. The signals are normalized to the asymptotic state intensities.

$34 \%$-would not affect the goodness of the present simulation. Note that the average experimental recombination rate constants of the chlorine atoms in $\mathrm{Cl}_{2}, \mathrm{Ar}, \mathrm{N}_{2}$, and $\mathrm{CO}_{2}$ are 5.8, 1.2, 1.0, and $5.7 \times 10^{-32} \mathrm{~cm}^{6}$ molecule ${ }^{-2} \mathrm{~s}^{-1}$, respectively. ${ }^{7,8,11,13,16}$

The average radiative lifetimes of the $\mathrm{A}$ and $\mathrm{B}$ states have been known to be $15 \mathrm{~ms}$ and $305 \mu \mathrm{s}$, respectively. ${ }^{16,27}$ According to the kinetic model as described in the previous section and with all the available experimental data, there still remain six rate constant parameters to be determined: $Q_{\mathrm{B}}, Q_{\mathrm{A}}, k_{\mathrm{ex}}, \alpha$, $\gamma$, and $R_{\mathrm{B}} / R_{\mathrm{A}}$. Note that the average photon emission rates of the $\mathrm{A}$ and $\mathrm{B}$ states are all much slower than the other transient processes considered. In the simulation, the steady-state relative population ratio of each vibrational level should match the steady-state chemiluminescence spectra obtained in a previous report. ${ }^{12}$ The smooth curves of Figures 3 and 4 show the bestfitted simulation. Since there were always some minor pickups of the luminescence wings coming from nearby vibrational bands by the detection system, the smooth curves shown were the results of the full simulation which took the slit function of the monochromator and the neighboring emissions into account. The vibrational quantum numbers shown in the figures are just the dominant levels in the photon emission. The best-fitted parameters of the rate constants of neat chlorine system are shown in the first column of Table 1. According to the present extended mechanism, the apparent electronic quenching rate constant of the B state measured by the laser-induced fluorescence technique is decomposed into two components: the electronic quenching rate constant of the $\mathrm{B}$ state into the nonradiative states and the interelectronic vibrational exchange rate constant between the $\mathrm{A}$ and $\mathrm{B}$ states. Comparing the 
TABLE 1: Fitted Parameters for the Rate Constants of Chlorine, Argon, Nitrogen, and Carbon Dioxide

\begin{tabular}{|c|c|c|c|c|}
\hline fitted parameters & $\mathrm{Cl}_{2}$ & $\mathrm{Ar}$ & $\mathrm{N}_{2}$ & $\mathrm{CO}_{2}$ \\
\hline$Q_{\mathrm{B}} / 10^{-13} \mathrm{~cm}^{3}$ molecule ${ }^{-1} \mathrm{~s}^{-1}$ & $\leq 20$ & $\leq 0.2$ & $\leq 0.2$ & $\leq 0.01$ \\
\hline$Q_{\mathrm{A}} / 10^{-13} \mathrm{~cm}^{3}$ molecule ${ }^{-1} \mathrm{~s}^{-1}$ & $5.5 \pm 1$ & $0.03 \pm 0.01$ & $0.03 \pm 0.01$ & $0.09 \pm 0.01$ \\
\hline$k_{\mathrm{ex}} / 10^{-11} \mathrm{~cm}^{3}$ molecule ${ }^{-1} \mathrm{~s}^{-1}$ & $0.4 \pm 0.1$ & $\leq 0.01$ & $\leq 0.01$ & $\leq 1.0$ \\
\hline$\alpha / \mathrm{cm}^{-1}$ & $111 \pm 7$ & $76 \pm 7$ & $90 \pm 7$ & $156 \pm 18$ \\
\hline$\gamma / \mathrm{cm}^{-1}$ & $710 \pm 290^{a}$ & $348 \pm 140$ & $278 \pm 140^{a}$ & $452 \pm 240^{a}$ \\
\hline$R_{\mathrm{B}} / R_{\mathrm{A}}$ & $\leq 0.2$ & $0.2 \pm 0.1$ & $0.2 \pm 0.1$ & $0.03 \pm 0.01$ \\
\hline assumed values $^{b}$ & & & & \\
\hline$R_{\mathrm{A}} / 10^{-34} \mathrm{~cm}^{6}$ molecules ${ }^{-2} \mathrm{~s}^{-1}$ & 200 & 41 & 34 & 190 \\
\hline
\end{tabular}

${ }^{a}$ The steady-state low-pressure-limit luminescence quantum yields of the A and B states in these buffer gases are assumed to be the same as those in the $\mathrm{Ar}$ case, i.e., $4.3 \%{ }^{b}$ See text for details.
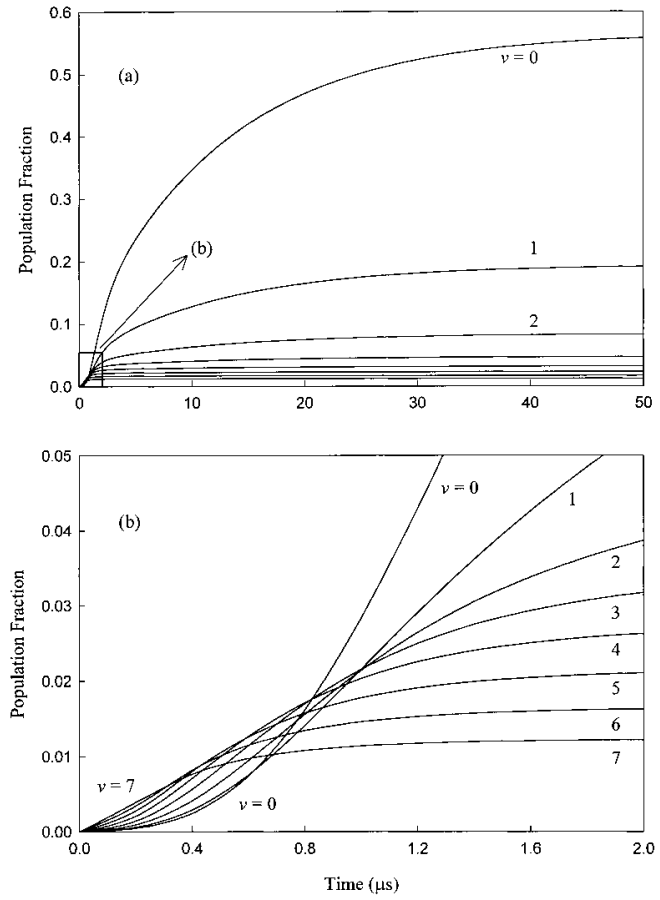

Figure 5. Theoretical transient vibrational populations at the lowest eight vibrational levels of the $\mathrm{B}$ state in neat $5.33 \mathrm{mbar}^{\mathrm{Cl}_{2}}$. The populations are normalized to the asymptotic state vibrational mole fractions.

measured electronic quenching rate constant $6.4 \times 10^{-12} \mathrm{~cm}^{3}$ molecule ${ }^{-1} \mathrm{~s}^{-1}$ and the upper bound of the electronic quenching rate constant of the $\mathrm{B}$ state into nonradiative states $2 \times 10^{-12}$ $\mathrm{cm}^{3}$ molecule ${ }^{-1} \mathrm{~s}^{-1}$, we found that the main population-depletion process for the $\mathrm{B}$ state is apparently the interelectronic exchange into the A state, which is energetically lower than that into the $\mathrm{B}$ state. Another interesting quantity is $\gamma$, which is a measure of the dispersion of the vibration population near the dissociation limit at the initial state of the recombination reactions. This quantity could be pinned down if the steady-state low-pressurelimit luminescence quantum yields of the A and B states in the atomic chlorine recombination reactions were known in the present mechanism. The only reported measurements were on the Ar system. By assuming that the dominant recombination process is through the energy-transfer mechanism and that the relative collision efficiencies among the recombination channels remain unchanged for different third bodies, ${ }^{28}$ the luminescence quantum yields of the chlorine system could be approximated by those of the argon system. The $\gamma$ value was then found to be $710 \pm 290 \mathrm{~cm}^{-1}$. In other words, the recombination reactions in the $\mathrm{Cl}_{2}$ buffer gas mainly populate the highest $710 \mathrm{~cm}^{-1}$ vibrational states near the dissociation limit.

Figure 5 shows the theoretical transient population fractions over the lowest eight vibrational states of the B state in 5.33
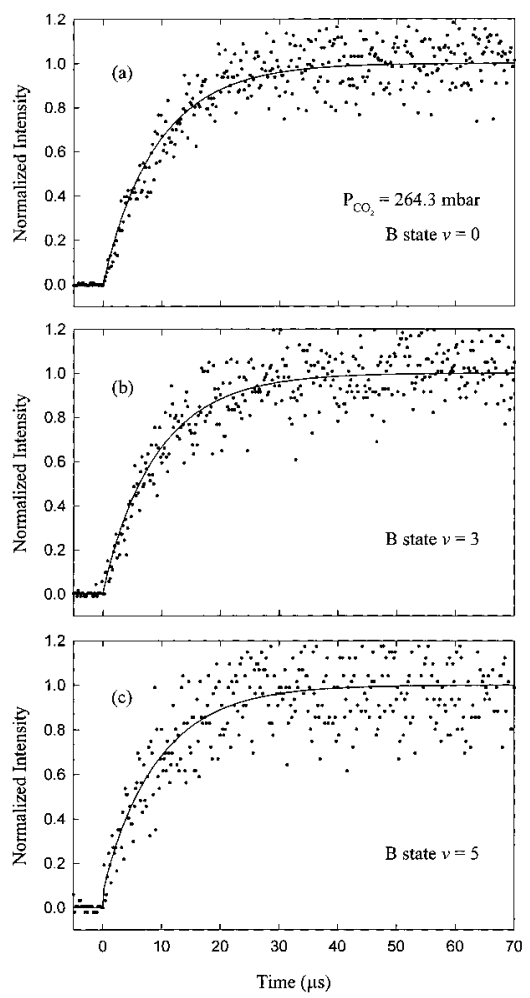

Figure 6. Transient recombination luminescence signals of $2.67 \mathrm{mbar}$ $\mathrm{Cl}_{2}$ in 264.3 mbar $\mathrm{CO}_{2}$ detected at the emissions originated from the $0,3(686.6 \mathrm{~nm}, \mathrm{~B}(v=3) \rightarrow \mathrm{X}(v=7))$, and 5 vibrational levels of the $\mathrm{B}$ state. Since there was always some scattered light recorded at time zero as the detection wavelength was shorter than $700 \mathrm{~nm}$, the scattering signal recorded on the laser-flashing channel has been reset to the background level in (b) and (c). The smooth curves are the full simulation of the corresponding experimental conditions. The signals are normalized to the asymptotic state intensities.

mbar $\mathrm{Cl}_{2}$ gas. As expected, owing to the initial preferential population of the recombined chlorine atoms into the higher vibrational states, the higher vibrational states shows higher population fractions in the very beginning of the recombination. After around $2 \mu \mathrm{s}$, the fast transient processes, which are the vibrational relaxation processes in the present case, more or less settle down to a steady-state situation. The following longtime rising is mainly controlled by the overall electronic quenching processes and shall be discussed in subsection $4 \mathrm{C}$.

4B. Buffer Mixtures with Argon, Nitrogen, and Carbon Dioxide. The vibrationally resolved transient luminescence of the chlorine atom recombination reactions was also measured in the buffer gases argon, nitrogen, and carbon dioxide. Figures 6 and 7 show the transient luminescence signals at 264.3 mbar carbon dioxide and 264.0 mbar nitrogen pressures, respectively. They are just two representatives in the experiments of the three 

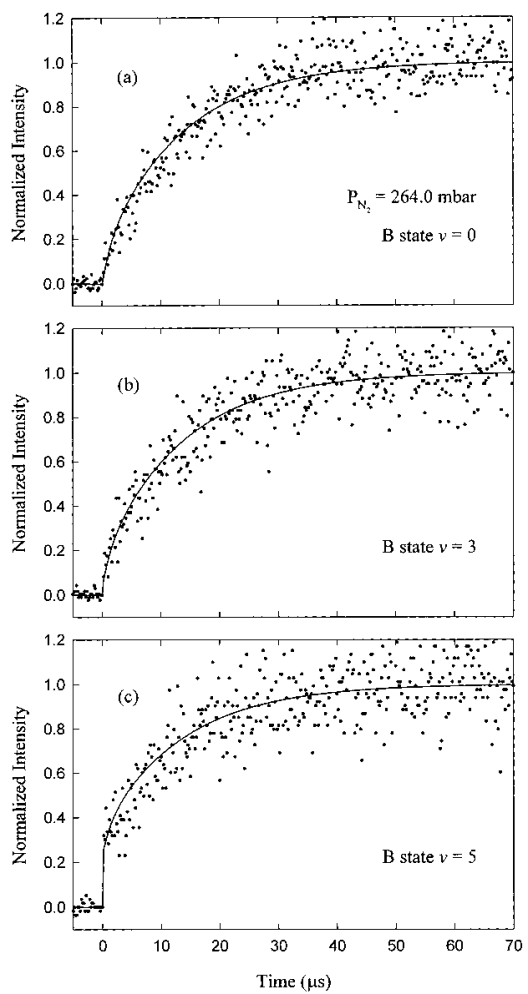

Figure 7. Transient recombination luminescence signals of $2.67 \mathrm{mbar}$ $\mathrm{Cl}_{2}$ in 264.0 mbar $\mathrm{N}_{2}$ detected at the emissions originated from the 0 , 3 , and 5 vibrational levels of the B state. The smooth curves are the full simulation of the corresponding experimental conditions. The signals are normalized to the asymptotic state intensities.

buffer gases. In all these measurements, the chlorine pressure was set at 2.67 mbar.

The master equations (10) and (11), which were explicitly expressed for the kinetics in a single buffer gas, could be easily expanded to include any additional number of buffer gases. The fitted kinetics parameters of the chlorine gas obtained in the neat chlorine system were adapted directly in the expanded master equations. Since the hard-sphere bimolecular collision rate constants between $\mathrm{Cl}_{2}$ and $\mathrm{Ar}, \mathrm{CO}_{2}$, and $\mathrm{N}_{2}$ are close to that between $\mathrm{Cl}_{2}$ itself, for these three buffer gases, the preexponential factor $V_{0}$ of the vibrational relaxation rate constant as expressed in eq 18 was set at the same value as that in the chlorine buffer gas. As suggested in the case of the neat chlorine system, the steady-state low-pressure-limit recombination luminescence quantum yields of the nitrogen and carbon dioxide buffer gases were also assumed to be the same as those of the argon buffer gas. The kinetics parameters of the extra buffer gases were then determined in the same way as described in the neat chlorine system. The smooth curves of Figures 6 and 7 are the best-fitted results for the vibrational levels and pressures studied in this report. Table 1 shows the best-fitted kinetics parameters of the three buffer gases.

As shown in Figure 6, at 264.3 mbar carbon dioxide pressure, the vibrational relaxation is much faster than the other processes such that the dominant transient behaviors over the observed vibrational levels are actually converging to a single rising curve. In other words, a steady-state situation was quickly set up among the vibrational states such that the observed transient behaviors were mainly due to the long-time decay of the system. For the other buffer gases, such as argon and nitrogen, the vibrational relaxation rate constants are much lower than that of the carbon dioxide gas such that, for these gases, steplike transient behaviors in the short-time domain were observed over the higher vibrational states under similar buffer pressures. Figure 7 shows a representative transient behavior in 264.0 mbar nitrogen gas. Similar behavior as Figure 7 was also observed with 264 mbar argon pressure or with a reduced 26.0 mbar carbon dioxide pressure. These suggest that the overall vibrational relaxation efficiencies of the nitrogen and argon gases are similar to each other and that of the carbon dioxide gas is more effective by about an order of magnitude.

As shown in Table 1, the A state quenching rate constant was determined to be $(3 \pm 1) \times 10^{-15} \mathrm{~cm}^{3}$ molecule ${ }^{-1} \mathrm{~s}^{-1}$ for both the argon and nitrogen gases. The quenching rate constants of the radiative states have been reported to be $(1.87 \pm 0.12)$ and $(2.66 \pm 0.04) \times 10^{-15} \mathrm{~cm}^{3}$ molecule ${ }^{-1} \mathrm{~s}^{-1}$ for the argon and nitrogen gases, respectively, in a series of high-pressure experiments. ${ }^{13,29}$ Despite of the huge buffer pressure difference in these two experiments, their measured results are in good agreement with each other. Note that the long-time decaying rate constant for the radiative $\mathrm{A}$ and $\mathrm{B}$ states is dominated by the quenching rate constant of the A state and shall be discussed in the following subsection. For the $\alpha$ values of the vibrational relaxation rate constant, the $\mathrm{CO}_{2}$ system has the largest value, followed by $\mathrm{Cl}_{2}$ and $\mathrm{N}_{2}$, and $\mathrm{Ar}$ has the smallest one. This order directly reflects the overall effectiveness of these buffer gases for the vibrational energy relaxation in the A and B states of the chlorine molecules. For the $\gamma$ values of the recombination reactions, under the approximation of the present report, the $\mathrm{Cl}_{2}$ system has the largest value, followed by $\mathrm{CO}_{2}$. The values of $\mathrm{Ar}$ and $\mathrm{N}_{2}$ are more or less close to each other. These values suggest that $\mathrm{Cl}_{2}$ is the most effective and $\mathrm{Ar}$ and $\mathrm{N}_{2}$ are the least effective in removing energy from the recombining chlorine atom pair during the recombination reactions among the four buffer gases. The recombination rate constant ratio between the B and A states is either equal to or less than 0.2. This is consistent with the conventional assumption that the major contribution to the recombination reactions is through the four bound states: $\mathrm{A}, \mathrm{A}^{\prime}, \mathrm{B}^{\prime}$, and $\mathrm{X} .{ }^{14}$ For the above general observations, to date there were no related reports available for comparisons. Further experimental and theoretical studies are needed to elucidate these phenomenological observations among the four buffer gases.

4C. The Long-Time Steady-State Behavior. The longtime steady-state behavior of the neat chlorine system shall be analyzed analytically and also numerically in this subsection. It is straightforward to extend it to multicomponent systems. Eqs 10 and 11 may be represented symbolically by the matrix form

$$
\frac{\mathrm{d}}{\mathrm{d} t}|X(t)\rangle=-\hat{L}|X(t)\rangle+\hat{R}|1\rangle
$$

in which $|X(t)\rangle$ is the vector of the vibrational state population, $|1\rangle$ is the unit vector, $\hat{R}$ is the recombination matrix, and $\hat{L}$ represents the remaining processes. For instance, the recombination matrix elements are

$$
\hat{R}_{i j}=R_{i} C^{2} M \delta_{i j}
$$

in which $R_{i}$ is the recombination rate constant into the $i$ th state, $C$ is the chlorine atom concentration, and $M$ is the third body concentration. If we define

$$
|Y(t)\rangle=|X(t)\rangle-\hat{L}^{-1} \hat{R}|1\rangle
$$




$$
\frac{\mathrm{d}}{\mathrm{d} t}|Y(t)\rangle=-\hat{L}|Y(t)\rangle
$$

The solution may be expressed in terms of the eigenfunctions $\left|y_{u}\right\rangle$ and eigenvalues $\lambda_{u}$ of $\hat{L}^{: 30}$

$$
|Y(t)\rangle=\sum_{u} a_{u} \exp \left(-\lambda_{u} t\right)\left|y_{u}\right\rangle
$$

in which, with the present initial condition $|X(0)\rangle=0$,

$$
a_{u}=\left\langle y_{u} \mid Y(0)\right\rangle=-\left\langle y_{u}\left|\hat{L}^{-1} \hat{R}\right| 1\right\rangle
$$

The final result is

$$
|X(t)\rangle=\sum_{u} a_{u} \exp \left(-\lambda_{u} t\right)\left|y_{u}\right\rangle+\hat{L}^{-1} \hat{R}|1\rangle
$$

Assuming that $0<\lambda_{1}<\lambda_{2}<\cdots$, for times longer than $\left(\lambda_{2}\right.$ $\left.-\lambda_{1}\right)^{-1}$, one obtains the steady-state solution

$$
|X(t)\rangle^{s s}=a_{1} \exp \left(-\lambda_{1} t\right)\left|y_{1}\right\rangle+\hat{L}^{-1} \hat{R}|1\rangle
$$

Eq 28 suggests that, under steady-state condition, the whole system and also its subsystems decay with the same rate, and the population ratio of any two subsystems is a constant. The smallest eigenvalue $\lambda_{1}$ could be related to the rate constants of eqs 10 and 11 as follows.

First, summing over all the vibrational states of eqs 10 and 11 , and at steady-state condition, the equation could be written as

$$
\begin{array}{r}
\frac{\mathrm{d}\left(\bar{A}^{\mathrm{ss}}+\bar{B}^{\mathrm{ss}}\right)}{\mathrm{d} t}=p-\left(D_{\mathrm{ss}}^{\mathrm{A}}+Q_{\mathrm{A}}+\frac{e_{\mathrm{A}}}{M}\right) M \bar{A}^{\mathrm{ss}}-( \\
D_{\mathrm{ss}}^{\mathrm{B}}+Q_{\mathrm{B}}+ \\
\left.\frac{e_{\mathrm{B}}}{M}\right) M \bar{B}^{\mathrm{ss}}
\end{array}
$$

in which $\bar{A}^{\mathrm{ss}}=\sum\left(A_{v}^{\infty}-A_{v}^{\mathrm{ss}}\right), \bar{A}_{v}^{\mathrm{ss}}=A_{v}^{\infty}-A_{v}^{\mathrm{ss}}=a_{v} e^{-\lambda_{1} t}, D_{\mathrm{ss}}^{\mathrm{A}}=$ $\left(\sum_{v} D_{\mathrm{v}}^{\mathrm{A}} \bar{A}_{v}^{\mathrm{ss}}\right) / \bar{A}^{\mathrm{ss}}$, and $e_{\mathrm{A}}$ is the average photon emission rate constant for the A state and similarly for the B state; the superscript notation $\infty$ means that the asymptotic value is taken, and the constant $p$ is

$$
\begin{array}{r}
p=\sum_{v}\left(D_{v}^{\mathrm{A}}+Q_{\mathrm{A}}+\frac{e_{\mathrm{A}}}{M}\right) A_{v}^{\infty} M+\sum_{u}\left(D_{u}^{\mathrm{B}}+Q_{\mathrm{B}}+\frac{e_{\mathrm{B}}}{M}\right) B_{u}^{\infty} M- \\
\left(R_{0}^{\mathrm{A}}+R_{0}^{\mathrm{B}}\right) M C^{2}
\end{array}
$$

Eq 29 could be further rearranged as

$$
\begin{array}{r}
\frac{\mathrm{d}\left(\bar{A}^{\mathrm{ss}}+\bar{B}^{\mathrm{ss}}\right)}{\mathrm{d} t}=p-\left[\left(D_{\mathrm{ss}}^{\mathrm{A}}+Q_{\mathrm{A}}+\frac{e_{\mathrm{A}}}{M}\right)\left(\frac{a}{a+b}\right)+\left(D_{\mathrm{ss}}^{\mathrm{B}}+\right.\right. \\
\left.\left.Q_{\mathrm{B}}+\frac{e_{\mathrm{B}}}{M}\right)\left(\frac{b}{a+b}\right)\right] \times M\left(\bar{A}^{\mathrm{ss}}+\bar{B}^{\mathrm{ss}}\right)
\end{array}
$$

in which $a=\sum a_{v}$, and $b=\sum b_{v}$. The smallest eigenvalue $\lambda_{1}$ is then just equal to the coefficient of the first-order term $\left(\bar{A}^{\text {ss }}+\right.$ $\bar{B}^{\text {ss) }}$ in eq 31 :

$$
\begin{array}{r}
\lambda_{1}=\left[\left(D_{\mathrm{ss}}^{\mathrm{A}}+Q_{\mathrm{A}}+\frac{e_{\mathrm{A}}}{M}\right)\left(\frac{a}{a+b}\right)+\left(D_{\mathrm{ss}}^{\mathrm{B}}+Q_{\mathrm{B}}+\frac{e_{\mathrm{B}}}{M}\right) \times\right. \\
\left.\left(\frac{b}{a+b}\right)\right] M
\end{array}
$$

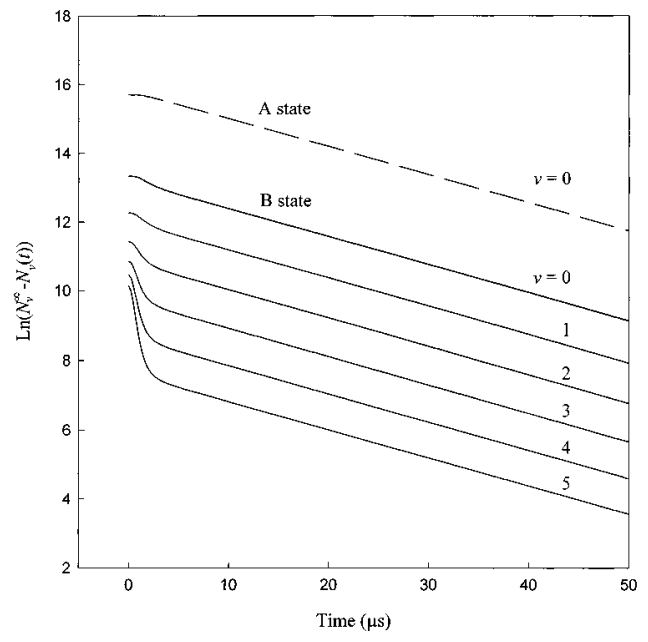

Figure 8. Theoretical decay behavior of the vibrational residual populations of the $\mathrm{A}$ and $\mathrm{B}$ states in neat $5.33 \mathrm{mbar} \mathrm{Cl}_{2}$. The long-time $\mathrm{Cl}_{2}$-concentration-normalized decay rate constant is $6.24 \times 10^{-13} \mathrm{~cm}^{3}$ molecule ${ }^{-1} \mathrm{~s}^{-1}$ for all the microstates.

Figure 8 shows the numerical residual populations over various vibrational states in the natural logarithm scale as the function time in the neat $5.33 \mathrm{mbar} \mathrm{Cl}_{2}$ system. Their long time steady-state residual populations are corresponding to the $\bar{A}_{v}^{\text {ss }}$ and $\bar{B}_{u}^{\text {ss }}$ values. With the input values of $Q_{\mathrm{A}}, Q_{\mathrm{B}}, e_{\mathrm{A}} / M$, and $e_{\mathrm{B}} / M$ of $5.5 \times 10^{-13}, 1.5 \times 10^{-12}, 5.1 \times 10^{-16}$, and $2.5 \times 10^{-13}$ $\mathrm{cm}^{3}$ molecule ${ }^{-1} \mathrm{~s}^{-1}$, respectively, the numerical long-time decay rate constant $\lambda_{1} / M$ is $6.24 \times 10^{-13} \mathrm{~cm}^{3}$ molecule ${ }^{-1} \mathrm{~s}^{-1}$. With the help of the numerical values of the steady-state populations, one obtained $a /(a+b)=0.932, b /(a+b)=0.068, D_{\mathrm{ss}}^{\mathrm{A}}=3.0$ $\times 10^{-15} \mathrm{~cm}^{3}$ molecule $\mathrm{s}^{-1}$, and $D_{\mathrm{ss}}^{\mathrm{B}}=2.1 \times 10^{-15} \mathrm{~cm}^{3}$ molecule ${ }^{-1} \mathrm{~s}^{-1}$. According to eq 32 , the analytical long-time decay rate constant becomes $6.2 \times 10^{-13} \mathrm{~cm}^{3}$ molecule ${ }^{-1} \mathrm{~s}^{-1}$, which is in agreement with the numerical value to within the numerical uncertainty. Of the total quenching rate constant, the quenching of the A state contributes $5.1 \times 10^{-13} \mathrm{~cm}^{3}$ molecule $\mathrm{e}^{-1} \mathrm{~s}^{-1}$ and that of the $\mathrm{B}$ state contributes the rest of $1.0 \times 10^{-13} \mathrm{~cm}^{3}$ molecule ${ }^{-1} \mathrm{~s}^{-1}$. The contribution from both the dissociation of the vibrational states into the atomic state and the photon emission processes is negligible under the present experimental conditions. Similar conclusion could also be reached if the upperbound value of $Q_{\mathrm{B}}$ was used in the above calculation.

\section{Conclusions}

The vibrationally resolved transient chemiluminescence of chlorine atom recombination reactions in chlorine, argon, nitrogen, and carbon dioxide buffer gases was studied by the laser photolysis/chemiluminescence detection method. With a full simulation of the experiments, either the rate constants of electronic quenching, vibrational relaxation within the same electronic states, and interelectronic vibrational energy transfer between the A and B states by the buffer gases were determined or their upperbound values were estimated. The long-time steady-state behavior of the mechanism was also analyzed and found to be dominated by the quenching processes under the present experimental conditions. The proposed extended mechanism for the chlorine atom recombination reactions could account for the related experimental measurements available in the literature and therefore resolve the inherent incompleteness in the traditionally simplified recombination mechanism. 
Acknowledgment. Financial support of this work by the National Science Council, Republic of China, is gratefully acknowledged.

\section{References and Notes}

(1) Bader, L. W.; Ogryzlo, E. A. J. Chem. Phys. 1964, 41, 2926.

(2) Hutton, E.; Wright, M. Trans. Faraday Soc. 1965, 61, 78.

(3) Clyne, M. A. A.; Coxon, J. A. Proc. R. Soc. A 1967, 298, 424. 816.

(5) Clyne, M. A. A.; Stedman, D. H. Trans. Faraday Soc. 1968, 64 , 2698.

(6) Browne, R. J.; Ogryzlo, E. A. J. Chem. Phys. 1970, 52, 5774.

(7) Widman, R. P.; DeGraff, B. A. J. Phys. Chem. 1973, 77, 1325.

(8) Hippler, H.; Troe, J. Int. J. Chem. Kinet. 1976, 8, 501. 704.

(9) Clyne, M. A. A.; Smith, D. J. J. Chem. Soc., Faraday II 1979, 75,

(10) Kernashitskii, L. A.; Nosenko, V. E.; Naumov, V. V.; Kochelap, V. A.; Izmailov, I. A. Chem. Phys. Lett. 1985, 116, 197.

(11) Weng, C.-J.; Ho, T.-I.; Su, T.-M. J. Phys. Chem. 1987, 91, 5235.

(12) Chang, L.-C.; Song, T.-T.; Tai, C.-C.; Su, T.-M. J. Phys. Chem. 1996, 100, 13548.

(13) Song, T.-T.; Su, T.-M., J. Phys. Chem. 1996, 100, 13554. 3860 .

(14) Song, T.-T.; Hwang, Y.-S.; Su, T.-M., J. Phys. Chem. 1997, 101,

(15) Hwang, M.-D.; Jiang, R.-C.; Su, T.-M. J. Chem. Phys. 1989, 91, 1626.

(16) Clyne, M. A. A.; Martinez, E. J. Chem. Soc., Faraday II 1980, 76, 1275.

(17) Coxon, J. A. J. Mol. Spectrosc. 1980, 82, 264.

(18) Ishiwata, T.; Ishiguro, A.; Obi, K. J. Mol. Spectrosc. 1991, 147, 300 .

(19) Le Roy, R. J. Molecular Spectroscopy; Barrow, R. F., Long, D. A., Millen, D. J., Eds.; Specialist Periodical Reports, The Chemical Society: London, 1973; Vol. 1, p 113.

(20) Clarke, A. G.; Burns, G. J. Chem. Phys. 1971, 55, 4717

(21) Clarke, A. G.; Burns, G. J. Chem. Phys. 1972, 56, 4636.

(22) Troe, J. J. Chem. Phys. 1977, 66, 4745.
(23) Hippler, H.; Troe, J. In Bimolecular Collisions; Baggott, J. E.; Ashfold, M. N., Eds.; Royal Society of Chemistry: London, 1989; p 209.

(24) Clyne, M. A. A.; McDermid, I. S. J. Chem. Soc., Faraday II 1979, $75,1313$.

(25) Bachmann, R.; Li, X.; Ottinger, Ch.; Vilesov, A. F.; Wulfmeyer, V. J. Chem. Phys. 1993, 98, 8606.

(26) The NAG Fortran Library, NAG Ltd., Oxford, United Kingdom, 1991.

(27) Coxon, J. A. Molecular Spectroscopy; Barrow, R. F., Long, D. A., Millen, D. J., Eds.; Specialist Periodical Reports, The Chemical Society: London, 1973; Vol. 1, p 177.

(28) For the reaction mechanisms of the recombination reactions of the halogen atoms, up to date, the available reports which specifically addressed the quantitative aspects of the reaction mechanisms were those of the bromine and iodine systems studied by the classical trajectory calculations. As suggested by the results of these studies, for the $\mathrm{Br}_{2} / \mathrm{Ar}$ system at 300 K (Wong, W. H.; Burns, G. J. Chem. Phys. 1973, 59, 2974), the conventional energy transfer mechanism would contribute $56 \%$ to the overall recombination reactions if the $\mathrm{ArBr}$ bond energy was taken to be $0.3 \mathrm{kcal} / \mathrm{mol}$. According to the above paper, since the dynamical behavior of the quasidimers of the $\mathrm{ArBr}$ intermediate complex is similar to that of the quasidimers of the energy transfer mechanism, one could include their contribution to the energy transfer mechanism and find that more than $70 \%$ of the recombination reactions would belong to a generalized energy transfer mechanism, which was specifically designated as the triple collision mechanism in the report. Because the bond energies of $\mathrm{ArCl}, \mathrm{ArN}_{2}$, and $\mathrm{ArCO}_{2}$ are either comparable to or smaller than that of $\mathrm{ArBr}$, their recombination reactions are also expected to be dominated by the generalized energy transfer mechanism at $300 \mathrm{~K}$. On the other end, the bond energy of $\mathrm{Cl}_{3}$ was known to be approximately $1 \mathrm{kcal} / \mathrm{mol}$. It was reckoned that the contributions of the two mechanisms to the total recombination reactions of the $\mathrm{Cl} / \mathrm{Cl}_{2}$ system were comparable to each other. Note that the above $0.3 \mathrm{kcal} / \mathrm{mol} \mathrm{ArBr}$ bond dissociation energy employed in the trajectory calculations is in good agreement with the recommended value of $0.30 \mathrm{kcal} /$ mol in a more recent transport study of atomic bromine in argon (Hwang, M.-D.; Jiang, R.-C.; Su, T.-M. J. Chem. Phys. 1989, 91, 1626).

(29) Tai, C.-C.; Chang, L.-C.; Su, T.-M. Unpublished results.

(30) van Kampen, N. G. Stochastic Processes in Physics and Chemistry; Elsevier Science Publishers B. V.: Amsterdam, 1992; p 96. 